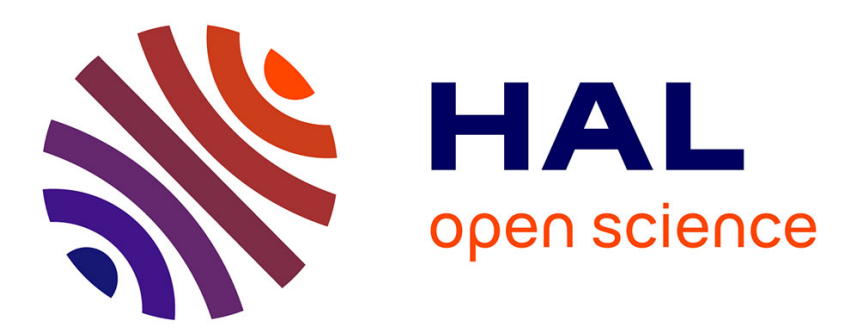

\title{
Sur une similitude entre des agrégats obtenus en émission ionique secondaire et des molécules observées en astrophysique \\ M. Leleyter
}

\section{- To cite this version:}

M. Leleyter. Sur une similitude entre des agrégats obtenus en émission ionique secondaire et des molécules observées en astrophysique. Journal de Physique Lettres, 1985, 46 (19), pp.915-922. 10.1051/jphyslet:019850046019091500 . jpa-00232918

\section{HAL Id: jpa-00232918 https://hal.science/jpa-00232918}

Submitted on 1 Jan 1985

HAL is a multi-disciplinary open access archive for the deposit and dissemination of scientific research documents, whether they are published or not. The documents may come from teaching and research institutions in France or abroad, or from public or private research centers.
L'archive ouverte pluridisciplinaire HAL, est destinée au dépôt et à la diffusion de documents scientifiques de niveau recherche, publiés ou non, émanant des établissements d'enseignement et de recherche français ou étrangers, des laboratoires publics ou privés. 
Classification

Physics Abstracts

$36.40-31.20 \mathrm{P}-98.40$

\title{
Sur une similitude entre des agrégats obtenus en émission ionique secondaire et des molécules observées en astrophysique
}

\author{
M. Leleyter (*) \\ Groupe de Physique Théorique, Faculté des Sciences, \\ 33, rue Saint-Leu, F-80039 Amiens Cedex, France
}

(Reçu le 16 avril 1985, révisé le 29 juin, accepté le 19 août 1985)

\begin{abstract}
Résumé. - On fait un rapprochement entre les molécules interstellaires $\mathrm{HC}_{\boldsymbol{x}} \mathrm{N}$ linéaires observées seulement pour $x$ impair et les ions $\mathrm{C}_{n} \mathrm{H}^{-}$obtenus en émission ionique secondaire qui sont beaucoup plus abondants pour $n$ pair que pour $n$ impair. Ces deux types d'amas étant isoélectroniques si $n=x+1$, l'étude des propriétés des ions $\mathrm{C}_{n} \mathrm{H}^{-}$par la méthode de Hückel permet d'obtenir également des résultats sur les molécules $\mathrm{HC}_{x} \mathrm{~N}$ observées dans les nuages interstellaires et de comprendre pourquoi on n'y trouve que celles de $x$ impair : en effet, le niveau de Fermi de ces chaînes se situe dans une bande de niveaux $\pi$ dégénérés et suivant la parité du nombre d'atomes de carbone, ce niveau est soit complet pour $n$ pair, donc $x$ impair (forte stabilité de l'amas), soit incomplet dans l'autre cas (stabilité beaucoup plus faible de l'amas). Le cas des ions $\mathrm{C}_{n} \mathrm{H}^{+}$et des molécules $\mathrm{C}_{n} \mathrm{H}$ est aussi considéré.
\end{abstract}

\begin{abstract}
A comparison is made between linear interstellar molecules $\mathrm{HC}_{\boldsymbol{x}} \mathrm{N}$ observed only for odd $x$ and $\mathrm{C}_{n} \mathrm{H}^{-}$ions obtained in SIMS experiments which are very much profuse for even $n$ than for odd ones. These two kinds of clusters are isoelectronic if $n=x+1$. So the study of the $\mathrm{C}_{n} \mathrm{H}^{-}$ ion properties with the Hückel method lets to get easily some results on $\mathrm{HC}_{\boldsymbol{x}} \mathrm{N}$ molecules and to understand why only the odd ones are detected in interstellar clouds : the Fermi level of these clusters indeed falls into a band of degenerated $\pi$ levels; so according to the carbon atom number parity, it is either full up for even $n$ or odd $x$ (great stability of the cluster) or half-filled in the other case (weaker stability of the corresponding cluster). $\mathrm{C}_{n} \mathrm{H}^{+}$ions and $\mathrm{C}_{n} \mathrm{H}$ molecules are considered too.
\end{abstract}

\section{Introduction.}

Les petits agrégats d'atomes constituent à l'heure actuelle un sujet de recherches en plein essor. Ils jouent en effet un rôle primordial dans un grand nombre de domaines tels que la catalyse, les aérosols, la nucléation et aussi l'astrophysique. C'est ainsi que les radioastronomes ont détecté ces dernières années dans les nuages interstellaires un certain nombre de molécules parmi lesquelles la famille des chaînes carbonées appelées parfois cyanopolyynes $\mathrm{HC}_{\boldsymbol{x}} \mathrm{N}$, et il se trouve

(*) Aussi Laboratoire de Physique des Solides, associé au CNRS, bât. 510, Université Paris-Sud, F-91405 Orsay Cedex, France. 
que jusqu'à présent, seules ont été identifiées les chaînes ayant un nombre impair d'atomes de carbone.

Outre les molécules de gaz cyanhydrique $\mathrm{HCN}$, on a ainsi mis en évidence les molécules $\mathrm{HC}_{x} \mathrm{~N}$ avec $x=3,5,7,9,11$ [1-4]. On a trouvé également les radicaux $\mathrm{CN}$ et $\mathrm{C}_{3} \mathrm{~N}$ ainsi que $\mathrm{CH}, \mathrm{C}_{2} \mathrm{H}$ et $\mathrm{C}_{4} \mathrm{H}[4,5] . \mathrm{C}_{3} \mathrm{H}$ a également été détecté très récemment mais avec une abondance d'environ un ordre de grandeur inférieure à celles de $\mathrm{C}_{2} \mathrm{H}$ et $\mathrm{C}_{4} \mathrm{H}$ [6]. On sait que toutes ces molécules interstellaires sont linéaires et que du fait de leur forte réactivité (puisque nombre d'entre elles sont des radicaux ou comportent des liaisons multiples), elles n'ont pu être mises en évidence au laboratoire que très récemment comme c'est le cas pour $\mathrm{C}_{2} \mathrm{H}$ [7], $\mathrm{C}_{4} \mathrm{H}$ et $\mathrm{C}_{3} \mathrm{~N}$ [8]. Et d'ailleurs aucun schéma généralement admis de formation des cyanopolyynes ne s'est encore vraiment dégagé $[9,10]$.

D'un autre côté, il existe des méthodes expérimentales permettant de produire des agrégats assez similaires comportant en particulier des chaînes de carbone. Ce sont les procédés d'émission ionique secondaire (E.I.S.) ou d'émission de sources à étincelles ou encore d'évaporation par chauffage ou par irradiation laser, méthodes bien connues où l'on peut observer l'apparition d'agrégats ionisés ou neutres tels que $\mathrm{C}_{n}^{+}, \mathrm{C}_{n}^{-}$ou $\mathrm{C}_{n}$, ou encore $\mathrm{C}_{n} \mathrm{H}^{+}, \mathrm{C}_{n} \mathrm{H}^{-}$[11].

En ce qui concerne les molécules $\mathrm{HC}_{x} \mathrm{~N}$, un raisonnement élémentaire sur les liaisons conjuguées dans une chaîne carbonée linéaire analogue à celui appliqué aux polyènes [12], permet de comprendre pourquoi les seules chaînes $\mathrm{HC}_{2 k+1} \mathrm{~N}$ sont susceptibles d'être observées. En effet, les molécules $H_{C_{x}} \mathbf{N}$ sont considérées par certains auteurs [13] comme ayant une structure linéaire du type polyacétylénique $\mathrm{H}-(\mathrm{C} \equiv \mathrm{C})_{p}-\mathrm{C} \equiv \mathrm{N}$, d'où leur nom de cyanopolyynes et il en est de même des radicaux $\mathrm{C}_{n} \mathrm{H}$ qui peuvent s'écrire $(\mathrm{C} \equiv \mathrm{C})_{p}-\mathrm{H}$.

Or c'est uniquement lorsque le nombre total d'atomes pouvant donner lieu à des liaisons $\pi$ est pair que les couches électroniques des extrémités du type $-\mathrm{C} \equiv \mathrm{N}$ ou $-\mathrm{C} \equiv \mathrm{C}-\mathrm{H}$ peuvent se fermer, donc pour $\mathrm{HC}_{2 k+1} \mathrm{~N}$ ou $\mathrm{HC}_{2 k}$. Dans le cas de l'autre parité, on a affaire à des couches ouvertes.

Des calculs ab initio sur les géométries, les propriétés « à un électron » et les énergies d'atomisation des molécules $\mathrm{HC}_{\boldsymbol{x}} \mathrm{N}$ ont également été effectués [13-15], mais uniquement pour $x$ impair. Ces calculs ont le très grand intérêt de fournir pour les énergies d'atomisation de ces molécules des valeurs absolues.

Cependant le raisonnement simple sur les liaisons, ou les calculs ab initio déjà cités, ne donnent aucun renseignement sur les chaînes $\mathrm{HC}_{2 k} \mathrm{~N}$, tant au point de vue de leur structure électronique et de la disposition de leurs niveaux d'énergie que de leurs énergies de dissociation, de sorte qu'il est donc intéressant de faire le calcul des stabilités relatives des chaînes $\mathrm{HC}_{x} \mathbf{N}$ ou $\mathrm{HC}_{n}$ quelle que soit la parité du nombre d'atomes, à l'aide d'un mode de calcul relativement simple, mais qui prend en compte la structure électronique des molécules ou radicaux indépendamment du fait qu'elles soient à " couche ouverte » ou à " couches fermées ».

Nous avons ainsi étudié, après Pitzer et Clementi [16], la structure et les propriétés électroniques des chaînes $C_{n}$ et de leurs ions [17]. Plus récemment, Douglas [2] a aussi fait appel à ce modèle pour discuter de la présence possible des chaînes $C_{n}$ de $n$ impair dans les nuages interstellaires. Nous avons également fait des calculs sur les chaînes $\mathrm{C}_{n} \mathrm{H}$ à partir de nos résultats expérimentaux [17] obtenus, rappelons-le, en bombardant une cible de graphite par des ions de gaz rares, en l'occurrence l'argon. Ces résultats permettent dans une certaine mesure de comprendre pourquoi en radioastronomie on n'a encore trouvé que les seules chaînes $\mathrm{HC}_{2 k+1} \mathrm{~N}$, et aussi d'étudier quelques propriétés de ces agrégats.

En effet, l'atome neutre d'azote comporte 5 électrons de valence. Les chaînes $\mathrm{HC}_{\boldsymbol{x}} \mathrm{N}$ se trouvent donc être isoélectroniques des agrégats $\left(\mathrm{HC}_{x+1}\right)^{-}$. Or on sait que les propriétés des molécules isoélectroniques sont extrêmement voisines. En partant de cette constatation simple, on peut donc induire à partir de nos propres résultats sur les $\mathrm{C}_{n} \mathrm{H}^{-}$, un certain nombre de caractéristiques des molécules $\mathrm{HC}_{\boldsymbol{x}} \mathrm{N}$. Remarquons cependant que, contrairement aux calculs ab initio cités plus haut, notre méthode de calcul, comme toute méthode fondée sur l'approximation de Hückel, 
ne peut donner que des valeurs relatives des grandeurs énergétiques considérées, fonctions d'un ou plusieurs paramètres, qui peuvent ensuite être fixés par comparaison à l'expérience ou à des calculs plus élaborés tels les calculs $a b$ initio.

\section{Résultats relatifs aux agrégats $C_{n} H$.}

2.1 EXPéRIENCES. - En ce qui concerne les résultats expérimentaux déjà publiés antérieurement [17, 18], rappelons seulement que les ions $\mathrm{C}_{n} \mathrm{H}^{+}$sont émis avec une fréquence plus grande quand $n$ est impair, et que au contraire, les ions négatifs $\mathrm{C}_{n} \mathrm{H}^{-}$sont beaucoup plus intenses (d'au moins un ordre de grandeur) si $n$ est pair. Le phénomène est ainsi bien plus net pour les ions négatifs que pour les ions positifs et il est tout à fait analogue aux alternances trouvées respectivement dans l'émission des ions homonucléaires de carbone $\mathrm{C}_{n}^{-}$et $\mathrm{C}_{n}^{+}$.

2.2 MéthOde De CALCUL. - Rappelons-la brièvement. Elle est essentiellement fondée sur l'approximation de Hückel dans laquelle on fait intervenir l'hybridation possible des atomes de carbone $[17,18]$. On sait que cette hybridation des orbitales $\mathbf{s}$ et $\mathbf{p}$ de l'atome de carbone peut être de 3 types : $\mathrm{sp}^{3}$ (carbone diamant « tétraédrique »), $\mathrm{sp}^{2}$ (carbone graphite avec par atome 3 orbitales hybrides coplanaires à $120^{\circ}$ les unes des autres) et $\mathrm{sp}$ (forme linéaire) qui est l'hybridation à laquelle nous nous attacherons ici.

Avant de traiter le cas simple de l'hybridation sp, donnons un aperçu du processus général de calcul. L'hamiltonien de Hückel avec hybridation se sépare entre hamiltonien des liaisons $\sigma$ et hamiltonien des liaisons $\pi$, suivant la terminologie habituelle, indépendants du fait qu'il s'agit d'une théorie «à un électron».

L'hamiltonien $\sigma$ se caractérise par l'existence, outre de la quantité $\beta_{\sigma}$ qui est l'intégrale de transfert (ou encore intégrale de résonance) comme dans toute méthode de Hückel [12], d'une deuxième grandeur $\Delta_{\sigma}$ qui est l'intégrale dite de promotion, spécifique de la théorie de Hückel avec hybridation [17-19]. Si on désigne par $E_{\mathrm{s}}$ et $E_{\mathrm{p}}$ les énergies atomiques des niveaux $\mathrm{s}$ et $\mathrm{p}$ du carbone et par $v$ le type de l'hybridation sp $\mathrm{sp}^{v}$ on a $\Delta_{\sigma}=\left(E_{\mathrm{s}}-E_{\mathrm{p}}\right) /(v+1)$. Dans le cas présent $E_{\mathrm{s}}=-19,45 \mathrm{eV}, E_{\mathrm{p}}=-10,74 \mathrm{eV}$ et $\beta_{\sigma}=-7,03 \mathrm{eV}$ [17].

L'hamiltonien $\pi$ qui est l'opérateur habituel de la méthode de Hückel, fait intervenir aussi une intégrale de transfert désignée ici par $\beta_{\pi}=-3,07 \mathrm{eV}$ [17]. Les niveaux $\pi$, qui n'existent que pour $v=1$ ou 2 , forment une bande centrée sur l'énergie atomique $E_{\mathrm{p}}$.

A partir de ces hypothèses, notre procédé de calcul a alors été le suivant pour les amas homonucléaires de carbone. Nous avons écrit un programme qui construit d'abord la matrice de Hückel avec hybridation pour les liaisons $\sigma$, la diagonalise et fournit ainsi les niveaux d'énergie $\sigma$, puis la matrice des liaisons $\pi$ sur laquelle il effectue le même travail. Ensuite tous ces niveaux sont classés par ordre d'énergie croissante, puis peuplés par les électrons de valence de la molécule ou de l'ion considérés, d'où l'on déduit alors la valeur de l'énergie électronique totale de l'amas.

C'est ainsi que dans le cas de l'hybridation sp, les niveaux des chaînes homonucléaires $\mathrm{C}_{n}$ ont une disposition tout à fait analogue à celle, bien connue, de Pitzer et Clementi [16], et qui est la suivante (Fig. 1) :

- une bande liante de $(n-1)$ états $\sigma$ remplis avec $2 n-2$ électrons,

- 2 niveaux de bout de chaîne $\sigma_{\mathrm{t}}$ situés de part et d'autre de la valeur $E_{\mathrm{m}}=\left(E_{\mathrm{s}}+E_{\mathrm{p}}\right) / 2$, également remplis (4 électrons),

- $n$ niveaux $\pi$ doublement dégénérés centrés sur $E_{\mathrm{p}}$ comme on l'a indiqué plus haut, et partiellement remplis,

- une bande antiliante de $(n-1)$ états $\sigma$ vides.

En fait, dans le cas simple de la chaîne linéaire (hybridation sp), les études faites précédemment [17] montrent que les seuls électrons $\pi$ suffisent à rendre compte des effets d'alternances observés sur les agrégats de carbone. Ils jouent en effet un rôle prépondérant du fait de la double dégéné- 


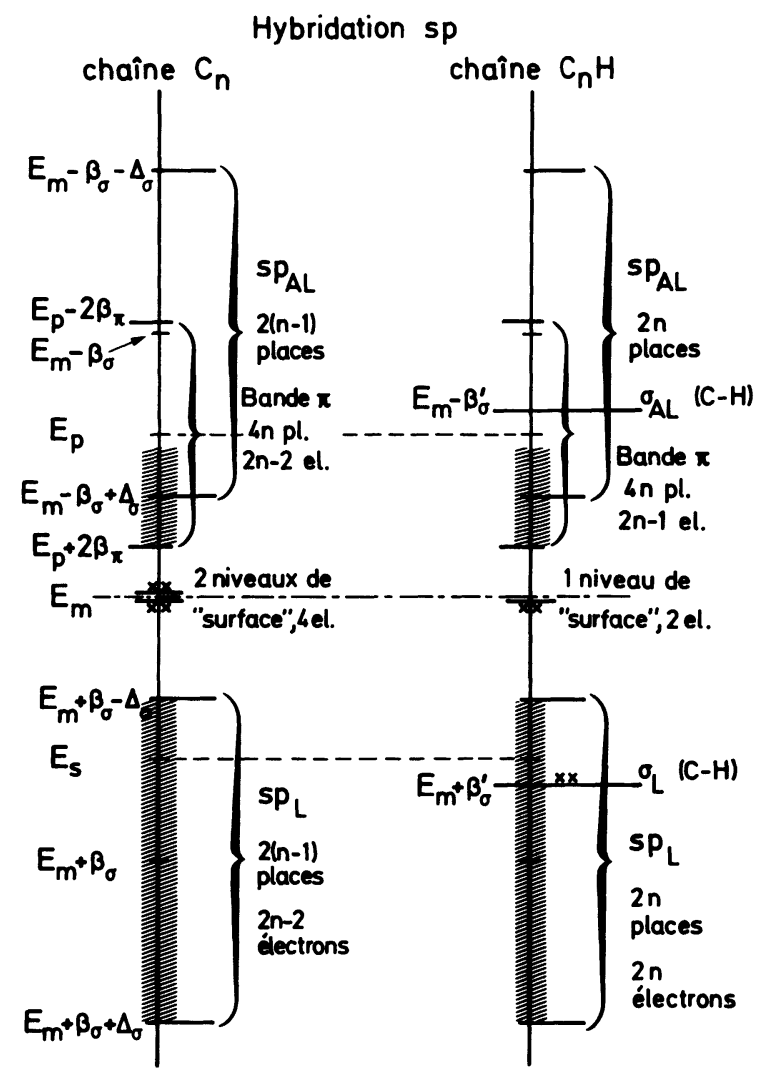

Fig. 1. - Niveaux d'énergie en hybridation sp des chaînes linéaires $C_{n}$ et $C_{n} H$. En hachures, les niveaux occupés : bande sp liante $\left(\mathrm{sp}_{\mathrm{L}}\right)$, une partie de la bande $\pi$ et les niveaux de surface $\sigma_{\mathrm{t}}$ dont l'occupation est indiquée par le nombre de croix. La population des bandes est également mentionnée. $C_{n}$ comporte 2 niveaux de surface voisins de $E_{\mathrm{m}}$. Pour $\mathrm{C}_{n} \mathrm{H}$, l'un des niveaux $\sigma_{\mathrm{t}}$ donne naissance dans la liaison C-H à un niveau $\sigma$ liant $\left(\sigma_{\mathrm{L}}\right)$ doublement occupé et un niveau $\sigma$ antiliant $\left(\sigma_{\mathrm{AL}}\right)$ vide.

[Energy levels in sp hybridization of linear chains $\mathrm{C}_{n}$ and $\mathrm{C}_{n} \mathrm{H}$. The occupied levels are hatched : the bonding $\mathrm{sp}$ band $\left(\mathrm{sp}_{\mathrm{L}}\right)$, a part of the $\pi$ band and the surface levels $\sigma_{\mathrm{t}}$ (the number of crosses shows their occupation). The electronic population of the bands is given too. $C_{n}$ has 2 surface levels close to $E_{\mathrm{m}}$. One of the $\sigma_{\mathrm{t}}$ levels of $\mathrm{C}_{n} \mathrm{H}$ gives rise, in the $\mathrm{C}-\mathrm{H}$ bond, to a bonding $\sigma$ level $\left(\sigma_{\mathrm{L}}\right)$ which accomodates 2 electrons and an antibonding $\sigma$ level $\left(\sigma_{\mathrm{AL}}\right)$ which is empty.]

rescence des niveaux $\pi$ qui augmente donc le poids relatif de ces niveaux. Pour la suite de cette discussion, nous nous bornerons donc à ne considérer que les électrons $\pi$. Mais il nous faudra cependant déterminer la structure de la bande liante afin de connaître le nombre des électrons $\pi$ mis en cause, nombre qui est la grandeur essentielle pour comprendre la structure électronique des agrégats étudiés.

2.3 StruCtURe ÉleCtroniQue DeS CHAÎNeS $\mathrm{C}_{n} \mathrm{H}$. - Dans le cas des chaînes hétéronucléaires $\mathrm{C}_{n} \mathrm{H}$, on suppose que l'atome d'hydrogène se lie en bout de chaîne de l'amas $\mathrm{C}_{n}$. L'hamiltonien $\sigma$ comporte alors, outre un nouveau terme diagonal dû au niveau s de l'atome $\mathrm{H}$, un terme supplémentaire faisant intervenir une nouvelle quantité $\beta_{\sigma}^{\prime}$ qui est l'intégrale de transfert relative 
à la liaison $\mathrm{C}-\mathrm{H}$. En fin de compte, tout se passe comme si le niveau de bout de chaîne $\sigma_{\mathrm{t}}$ adjacent de l'atome d' "impureté » $\mathrm{H}$, en se combinant à un niveau de cet atome, donnait 2 autres niveaux, l'un liant $\sigma_{\mathrm{L}}(\mathrm{C}-\mathrm{H})\left(\right.$ Fig. 1) situé à $\beta_{\sigma}^{\prime}$ de $E_{\mathrm{m}}$ et l'autre antiliant $\sigma_{\mathrm{AL}}(\mathrm{C}-\mathrm{H})$ situé à $-\beta_{\sigma}^{\prime}$ de $E_{\mathrm{m}}$. De sorte que l'on a finalement par ordre d'énergie croissante :

- une bande liante de $n$ états $\sigma$ (les $n-1$ de $\mathrm{C}_{n}+$ le niveau $\sigma_{\mathrm{L}}(\mathrm{C}-\mathrm{H})$ ) remplis par $2 n$ électrons,

- un seul niveau dit «de surface» $\sigma_{\mathrm{t}}$ rempli, correspondant à l'extrémité encore libre de la chaîne $\mathrm{C}_{n}$,

- $n$ niveaux $\pi$ doublement dégénérés, partiellement remplis,

- une bande antiliante de $n$ états $\sigma$ vides (les $n-1$ de $\mathrm{C}_{n}+\sigma_{\mathrm{AL}}(\mathrm{C}-\mathrm{H})$ ).

Les chaînes neutres $\mathrm{C}_{n} \mathrm{H}$ ont en tout $4 n+1$ électrons de valence dont $2 n-1$ électrons $\pi$ en tenant compte du remplissage des bandes détaillées ci-dessus. Cela revient en fait à assimiler la liaison $\mathrm{C}-\mathrm{H}$ à une liaison $\mathrm{C}-\mathrm{C}$, approximation supplémentaire que nous avons déjà faite plusieurs fois. La présence de l'atome d'hydrogène se traduit simplement, outre les 2 niveaux $\sigma_{\mathrm{L}}(\mathrm{C}-\mathrm{H})$ et $\sigma_{\mathrm{AL}}(\mathrm{C}-\mathrm{H})$, par l'existence d'un électron de valence supplémentaire.

Dans ces conditions, les ions $\mathrm{C}_{n} \mathrm{H}^{+}$comportent donc $2 n-2$ électrons $\pi$ et les ions $\mathrm{C}_{n} \mathrm{H}^{-}$ en comportent $2 n$. Dans l'hybridation sp, les niveaux $\pi$ sont, comme nous l'avons signalé plus haut, doublement dégénérés. De ce fait, le dernier niveau $\pi$ occupé (niveau de Fermi de l'amas ou « HOMO » (highest occupied molecular orbital)) contient suivant la parité de $n$, soit 2 , soit 4 électrons (niveau complet). Dans ce dernier cas, on a donc une " couche complète " qui correspond alors à un agrégat dont la stabilité est toujours plus forte (comme on l'a rappelé en introduction) que celle d'un agrégat qui correspond à un niveau seulement à moitié rempli.

Il en résulte que les ions $\mathrm{C}_{n} \mathrm{H}^{-}$ont un niveau de Fermi complet si $n$ est pair, tandis que cela se produit pour les ions $\mathrm{C}_{n} \mathrm{H}^{+}$si $n$ est impair.

Si l'on considère les valeurs des énergies électroniques totales des électrons $\pi, D_{e}^{q}(n)$ (avec $q=0,+1$ ou -1 suivant qu'il s'agit des amas neutres ou des ions positifs ou négatifs), exprimées en unités $\left|\beta_{\pi}\right|$ [20], ou plutôt les valeurs des accroissements $\Delta_{n}^{q}$ de ces énergies électroniques $\pi$ définis par $\Delta_{n}^{q}=D_{e}^{q}(n)-D_{e}^{q}(n-1)$ qui correspondent à la quantité d'énergie électronique gagnée par l'amas de $n-1$ atomes de carbone quand on lui ajoute un atome de carbone supplémentaire (donc une liaison $\mathrm{C}-\mathrm{C}$ de plus) (Tableau I), on trouve effectivement des oscillations de $\Delta_{n}^{q}$ avec la parité de $n$. Les accroissements sont ainsi bien plus forts pour les ions négatifs de $n$ pair (le gain d'énergie est plus grand quand on passe d'un ion de rang $n-1$ impair à un ion de rang $n$ pair que dans le cas inverse) et pour les ions positifs de $n$ impair comme le laissait prévoir la discussion précédente sur le remplissage du niveau de Fermi. Remarquons aussi que l'écart entre les valeurs fortes et les valeurs faibles des $\Delta_{n}^{+}$ou $\Delta_{n}^{-}$, considérable pour $n$ petit, s'amenuise de plus en plus au fur et à mesure que la chaîne carbonée s'allonge, ce qui est normal puisque l'augmentation du nombre des niveaux d'énergie avec $n$ conduit à la diminution de leurs écarts respectifs donc de ceux des $\Delta_{n}$.

Le phénomène d'oscillations des $\Delta_{n}$ est beaucoup moins marqué pour les chaînes neutres du fait que le niveau de Fermi comporte alors soit un seul, soit 3 électrons $\pi$, de sorte qu'il ne peut jamais être complet et que la stabilité des amas est donc plus faible; mais on peut cependant observer que les $\Delta_{n}$ sont légèrement plus forts pour les valeurs paires de $n$, donc que les chaînes neutres $\mathrm{C}_{n} \mathrm{H}$ sont plus stables si $n$ est pair.

Dans le tableau I, nous avons mentionné aussi $\mathrm{PI}_{n}$ et $\mathrm{AE}_{n}$ qui sont les énergies d'ionisation et les affinités électroniques des chaînes $\mathrm{C}_{n} \mathrm{H}$ obtenues simplement à partir du théorème de Koopmans et en tenant compte de ce que $E_{\mathrm{p}}=3,50 \beta_{\pi}$. On voit que les $\mathrm{PI}_{n}$ et les $\mathrm{AE}_{n}$ sont plus élevés pour $n$ pair ; les $\mathrm{C}_{n} \mathrm{H}$ seront donc plus faciles à ioniser positivement si $n$ est impair, à ioniser négativement si $n$ est pair puisque dans ce cas l'énergie gagnée en passant de $\mathrm{C}_{n} \mathrm{H}$ à $\mathrm{C}_{n} \mathrm{H}^{-}$est plus forte. Les variations des $\mathrm{PI}_{n}$ et $\mathrm{AE}_{n}$ agissent dans le même sens (pour renforcer la même 
Tableau I. - Agrégats $\mathrm{C}_{n} \mathrm{H}$ (neutres ou ionisés) linéaires (hybridation sp) avec $\mathrm{H}$ en bout de chaîne étudiés dans l'approximation de Hückel. Les grandeurs (exprimées en unités $\left.\left|\beta_{\pi}\right|\right)$ sont définies dans le texte. Les valeurs les plus fortes des $\Delta_{n}^{q}$ (correspondant donc aux amas les plus stables) sont en italiques.

[Linear $\mathrm{C}_{n} \mathrm{H}$ clusters (neutral or ionized) (hybridization sp) with $\mathrm{H}$ at the end of the $\mathrm{C}_{n}$ chain, studied in the Hückel approximation. The quantities (in $\left|\beta_{\pi}\right|$ units) are defined in the text. The largest values of the $\Delta_{n}^{q}$ (which correspond to the most stable clusters) are in italics.]

\begin{tabular}{|c|c|c|c|c|c|c|c|c|}
\hline$\frac{\mathrm{C}_{n} \mathrm{H}}{n}$ & $-D_{\mathrm{e}}^{+}(n)$ & $-\Delta_{n}^{+}$ & $\mathrm{PI}_{n}$ & $-D_{\mathrm{e}}(n)$ & $-\Delta_{n}$ & $-\mathrm{AE}_{n}$ & $-D_{\mathrm{e}}^{-}(n)$ & $-\Delta_{n}^{-}$ \\
\hline 1 & 0 & - & 3,50 & 0 & - & 3,50 & 0 & - \\
2 & 2,00 & 2,00 & 4,50 & 3,00 & 3,00 & 4,50 & 4,00 & 4,00 \\
3 & 5,66 & 3,66 & 3,50 & 5,66 & 2,66 & 3,50 & 5,66 & 1,66 \\
4 & 7,71 & 2,05 & 4,12 & 8,33 & 2,67 & 4,12 & 8,94 & 3,29 \\
5 & 10,93 & 3,22 & 3,50 & 10,93 & 2,60 & 3,50 & 10,93 & 1,98 \\
6 & 13,09 & 2,16 & 3,94 & 13,53 & 2,60 & 3,94 & 13,98 & 3,05 \\
7 & 16,11 & 3,02 & 3,50 & 16,11 & 2,57 & 3,50 & 16,11 & 2,13 \\
8 & 18,34 & 2,24 & 3,85 & 18,69 & 2,59 & 3,85 & 19,03 & 2,92 \\
9 & 21,25 & 2,91 & 3,50 & 21,25 & 2,56 & 3,50 & 21,25 & 2,22 \\
10 & 23,55 & 2,30 & 3,78 & 23,83 & 2,58 & 3,78 & 24,11 & 2,85 \\
11 & 26,38 & 2,83 & 3,50 & 26,38 & 2,55 & 3,50 & 26,38 & 2,28 \\
12 & 28,70 & 2,32 & 3,74 & 28,94 & 2,56 & 3,74 & 29,18 & 2,80 \\
\hline
\end{tabular}

parité) que les stabilités représentées par les valeurs des quantités $\Delta_{n}^{q}$ et sont donc ici en accord avec le comportement expérimental en E.I.S. (ce qui n'est pas toujours le cas, mais se traduit dans le cas présent par des alternances très importantes).

\section{Comparaison avec les molécules interstellaires et discussion.}

Si l'on revient à présent aux chaînes $H_{C_{x}} \mathbf{N}$ repérées dans des nuages interstellaires uniquement pour $x$ impair, et qui sont donc isoélectroniques des ions $\mathrm{HC}_{2 k}^{-}(2 k=x+1)$, le calcul effectué plus haut par la méthode de Hückel peut donc encore s'y appliquer si, comme nous l'avons dit en introduction, on assimile l'atome d'azote au point de vue structure électronique externe à un ion négatif $\mathrm{C}^{-}$.

Notre calcul a montré que les stabilités des ions $\mathrm{C}_{n} \mathrm{H}^{-}$, représentées par les quantités $\Delta_{n}^{-}$, sont beaucoup plus fortes pour $n$ pair que pour $n$ impair. Les affinités électroniques qui présentent les mêmes alternances, peuvent également agir pour renforcer aussi les ions de $n$ pair.

On voit donc que le simple calcul utilisant la méthode de Hückel permet de comprendre pourquoi jusqu'à présent les seules molécules $\mathrm{HC}_{2 k+1} \mathrm{~N}$ ont été observées. Elles sont en effet beaucoup plus stables, donc plus faciles à détecter parce que présentes en grandes quantités dans les nuages interstellaires, que les $\mathrm{HC}_{2 k} \mathrm{~N}$ qui, si elles existent, sont en quantités encore trop faibles pour être décelées avec les moyens actuels d'investigation.

En ce qui concerne les radicaux $\mathrm{C}_{2} \mathrm{H}, \mathrm{C}_{3} \mathrm{H}$ et $\mathrm{C}_{4} \mathrm{H}$, identifiés également dans des nuages interstellaires [4-6], notre calcul montre que les chaînes $\mathrm{C}_{2 k} \mathrm{H}$ sont plus stables que les chaînes $\mathrm{C}_{2 k+1} \mathrm{H}$. Notre modèle simple dans l'approximation de Hückel permet donc de comprendre aussi la concentration très faible du radical $\mathrm{C}_{3} \mathrm{H}$ dans les nuages interstellaires. Notons par ailleurs que les radicaux $C N$ et $C_{3} N$ sont isoélectroniques respectivement de $\mathrm{C}_{2}^{-}$et $\mathrm{C}_{4}^{-}$qui sont 
eux-mêmes beaucoup plus stables que $\mathrm{C}^{-}, \mathrm{C}_{3}^{-}$ou $\mathrm{C}_{5}^{-}$d'après les conclusions d'articles précédents [17]. Cette dernière remarque est en accord avec le fait que l'on n'observe pas encore le radical $\mathrm{C}_{2} \mathrm{~N}$ isoélectronique de $\mathrm{C}_{3}^{-}$. Signalons que Friberg et al. [21] ont prédit certaines propriétés du radical $\mathrm{C}_{3} \mathrm{~N}$ en se fondant aussi sur le fait qu'il est isoélectronique de $\mathrm{C}_{4} \mathrm{H}$.

Par ailleurs, dans les cas des ions positifs $\mathrm{C}_{n} \mathrm{H}^{+}$, on peut observer que le résultat de notre calcul " statique ", c'est-à-dire qui consiste à évaluer la stabilité des molécules elles-mêmes sans faire appel à un mécanisme de formation, est le même que celui du calcul effectué d'un point de vue " dynamique » en étudiant la formation de ces mêmes ions au cours de diverses réactions chimiques qui font intervenir entre autres des molécules d'acétylène, par Mitchell et Huntress [22] qui trouvent les ions $\mathrm{C}_{n} \mathrm{H}_{m}^{+}$beaucoup plus abondants pour $n$ impair, quel que soit le nombre $m$ d'atomes d'hydrogène, donc en particulier pour $m=1$.

Signalons pour terminer que nous avons aussi étudié les chaînes linéaires $\mathrm{C}_{n} \mathrm{H}$ avec l'approximation CNDO et les principaux résultats confirment tout à fait ceux du calcul présenté ici pour les ions $\mathrm{C}_{n} \mathrm{H}^{q}(q=+1$ ou -1$)[18]$.

\section{Conclusion.}

Nous avons donc pu mettre en parallèle dans cet article les molécules interstellaires $\mathrm{HC}_{\boldsymbol{x}} \mathbf{N}$ et les ions secondaires $\mathrm{C}_{n} \mathrm{H}^{-}$et expliquer ainsi le fait que les amas d'une certaine parité sont très renforcés. En outre, nous avons obtenu des valeurs des énergies d'ionisation $\mathrm{PI}_{n}$ et des affinités électroniques $\mathrm{AE}_{n}$ des agrégats $\mathrm{C}_{n} \mathrm{H}$, grandeurs qui présentent aussi des alternances avec la parité de $n$.

Les phénomènes d'alternances observés dans deux domaines aussi différents que les molécules existant dans les nuages interstellaires ou les agrégats ionisés obtenus en E.I.S. peuvent donc être interprétés par des calculs de stabilités analogues, même dans un modèle aussi simple que l'approximation de Hückel avec hybridation. Et il apparait une fois encore que ces oscillations observées sur les agrégats quel que soit leur mode de formation, sont les conséquences directes de leur structure électronique et en particulier du fait que le niveau de Fermi tombe dans une bande de niveaux $\pi$ dégénérés, et que son remplissage est donc directement lié à la parité du nombre d'atomes de carbone de l'agrégat considéré.

Nous avons rappelé en introduction les hypothèses récentes sur une structure de type polyacétylénique [13]; elles expliqueraient le fait que ces molécules interstellaires sont linéaires; cette structure est également considérée par Rohlfing et al. [23] qui, partant de ces mêmes hypothèses, postulent l'existence d'une nouvelle phase cristalline du carbone, la phase appelée "carbyne » autre que le graphite ou le diamant, seule à même d'interpréter l'émission d'agrégats de carbone d'une parité donnée sous bombardement laser d'une cible de graphite; cette phase serait donc constituée, comme les formules données en introduction l'indiquent, de chaînons d'acétylène reliés les uns aux autres et est supposée exister à haute température d'après l'étude de Rohlfing et al. [23]. Elle correspondrait donc à l'hybridation sp, alors que le graphite et le diamant représentent les deux autres types d'hybridation $\mathrm{sp}^{2}$ et $\mathrm{sp}^{3}$. Il faudrait alors étudier si cette phase " carbyne " peut également subsister dans les conditions de température et de pression des nuages interstellaires, totalement différentes de celles des expériences de Rohlfing et al.

Cette structure qui permet également d'expliquer en partie l'origine des différences de stabilité des chaînes avec la parité du nombre d'atomes de carbone, est tout à fait en accord avec le résultat de nos calculs, bien que nous ayons considéré pour simplifier que toutes les liaisons $\mathrm{C}-\mathrm{C}$ étaient équivalentes avec la même distance interatomique, donc le même $\beta_{\pi}$ [20]. Le fait que ces molécules soient linéaires est en outre une bonne confirmation du modèle simple d'étude des carbures que nous avons utilisé ici et qui nous avait servi auparavant à étudier divers carbures de métaux de transition $[24,25]$.

Une dernière remarque peut encore être faite au sujet des ions $\mathrm{C}_{n} \mathrm{H}^{+}$émis préférentiellement pour $n$ impair. Dans une étude précédente sur les carbures [25], nous avions signalé que l'élec- 
tronégativité de l' "impureté » ajoutée aux chaînes $C_{n}$ joue un rôle important quant au renforcement d'une parité donnée de $n$ : quand l'élément est fortement électronégatif, les ions de $n$ impair sont émis préférentiellement, alors que ce sont ceux de $n$ pair qui le sont si l'impureté est plutôt électropositive. On voit que dans le cas présent avec une électronégativité assez forte de l'hydrogène (de l'ordre de 2,20) [26], on a bien un renforcement des ions $\mathrm{C}_{n} \mathrm{H}^{+}$de $n$ impair.

\section{Remerciements.}

Nous remercions le rapporteur de cet article pour les nombreuses références qu'il nous a signalées ainsi que Monsieur le Professeur G. Berthier et Mme J. Baudet pour les conseils judicieux qu'ils nous ont donnés.

\section{Bibliographie}

[1] LeQueUX, J., La Recherche 164 (1985) 330.

[2] Douglas, A. E., Nature 269 (1977) 130.

[3] Bell, M. B., Feldman, P. A., Matthews, H. E., Astron. Astrophys. 101 (1981) L-13.

[4] Freeman, A., Miller, T. J., Nature 301 (1983) 402.

[5] Thaddeus, P., Philos. Trans. R. Soc. London A 303 (1981) 469.

[6] Johansson, L. E. B., Andersson, C., Elldèr, J., Friberg, P., Hualmarson, A., Hoglund, B., Irvine, W. M., Olofsson, H., RydBeck, G., Astron. Astrophys. 130 (1984) 227.

[7] Gottlieb, C. A., Gottlieb, E. W., Thaddeus, P., Astrophys. J. 264 (1983) 740.

[8] Gottlieb, C. A., Gottlieb, E. W., Thaddeus, P., Astrophys. J. 275 (1983) 916.

[9] Benson, P. J., Myers, P. C., Astrophys. J. 242 (1980) L-87.

[10] Leung, C. M., Herbst, E., Huebner, W. F., Astrophys. J. Suppl. 56 (1984) 231.

[11] Voir références dans JoYes, P., Leleyter, M., J. Physique 45 (1984) 1681.

[12] SALEM, L., The Molecular Orbital Theory of Conjugated Systems (New-York, Benjamin) 1966.

[13] HA, T. K., NGuYen, M. T., Z. Naturforschung, Teil A 37 (1982) 1272.

[14] Wilson, S., Green, S., Astrophys. J. 212 (1977) L87.

[15] Boyd, R. J., Jones, W. E., Ling, K. W., Chem. Phys. 58 (1981) 203.

[16] Pitzer, K. S., Clémenti, E., J. Am. Chem. Soc. 81 (1958) 4477.

[17] Leleyter, M., Joyes, P., J. Physique 36 (1975) 343.

[18] Leleyter, M., Thèse de Doctorat d'Etat (1975) Orsay.

[19] Friedel, J., Lannoo, M., J. Physique 34 (1973) 115.

Friedel, J., LanNoO, M., J. Physique 34 (1973) 483.

[20] Ceci implique que l'on suppose identiques toutes les liaisons $C-C$ de la chaine, avec une formule du type $=\mathrm{C}=\mathrm{C}=\mathrm{C}=\mathrm{au}$ lieu d'une succession alternée de liaisons simples et multiples comme le laisserait attendre la formule de type polyacétylénique.

[21] Friberg, P., Hualmarson, A., Irvine, W. M., Guélin, M., Astrophys. J. 241 (1980) L13.

[22] Mitchell, G. F., HunTress, W. T. Jr., Nature 278 (1979) 722.

[23] Rohlfing, E. A., Cox, D. M., Kaldor, A., J. Chem. Phys. 81 (1984) 3322.

[24] Leleyter, M., Ortoli, S., Joyes, P., Surface Sci. 106 (1981) 293.

[25] Leleyter, M., J. Physique 42 (1981) 1115.

[26] Gray, H. B., Electrons and Chemical Bonding (New-York, Benjamin) 1964, p. 69. 